\title{
Anestesia em Jacaré-americano (Alligator mississipiensis) para Amputação de Membro
}

\author{
Anesthesia in American Alligator (Alligator mississipiensis) for a Limb Amputation \\ Rochelle Gorczak' ${ }^{1}$, Rafael Oliveira Chaves ${ }^{2}$, Marcella Linhares Teixeira², Ítallo Barros de Freitas ${ }^{3}$, \\ Raimy Costa Martins' ${ }^{1}$ Marilia Avila Valandro4 ${ }^{4}$ Bruna Copat ${ }^{2} \&$ André Vasconcelos Soares²
}

\begin{abstract}
Background: The knowledge of secure anesthetic protocols for reptiles is very important, especially because they are aggressive species and they have peculiar physiology, unpredictable reactions to the same drug in different environmental conditions and different physiological responses can be observed. The anesthetic protocol aims immobilization, analgesia and muscle relaxation for adequate, safe and easily reversible anesthesia. Few reports have been described on anesthesia in animals and, before that, the objective of this study is to report the anesthetic protocol that was used and vital parameters monitoring in an Alligator mississipiensis that was submitted to surgery for limb amputation.

Case: A female American alligator was attended, with about 8-year-old, $2 \mathrm{~m}$ long and 268,964 pounds, with fight history with other animal six months ago, resulting in a wound in the right toracic limb. When the lesion occurred, it was the breeding season of the species, so we chose not to perform the treatment at that time. An X-ray of the member was performed, which showed, among other changes, osteomyelitis and septic arthritis. Thus, the limb amputation was indicated. Ketamine association $(10 \mathrm{mg} / \mathrm{kg})$ and medetomidine $(0.1 \mathrm{mg} / \mathrm{kg})$ were used as anestesic premedication, both administered intramuscularly. For anesthetic induction, propofol $(4 \mathrm{mg} / \mathrm{kg}$ ) intravenously was used. The animal was intubated using an endotracheal tube number 11 without inflating the cuffing, and for the maintenance it was employed isoflurane. The heart rate was measured using Doppler and the respiratory rate by visual and balloon observation in the oxygen circular system. The other parameters were measured using a multiparameter monitor sensor connected to the tongue. The local anesthetic block was made close to the incision line, the medium third humerus with $2 \mathrm{mg} / \mathrm{kg}$ of $2 \%$ lidocaine without vasoconstrictor, diluted in $0.9 \% \mathrm{NaCl}$ until the volume of $10 \mathrm{~mL}$ is completed to assist in analgesia. The parameters recorded during the procedure, which lasted $80 \mathrm{~min}$, remained within the normal pattern of species (three breaths per minute, 30 heart beats per min and $80.6^{\circ} \mathrm{F}$ temperature). After surgery meloxicam $(0.2 \mathrm{mg} / \mathrm{kg})$ and atipamezole reverser $(0.5 \mathrm{mg} / \mathrm{kg})$ were administered, both intramuscularly. The animal took about seven hours to fully return from anesthesia.

Discussion: Different anesthetics protocols may be employed to crocodilians using both local anesthetic techniques and general, in order to promote analgesia, muscle relaxation and adequate anesthesia. Still, it is very important to monitor the patient during the procedure, in order to be a different physiology kind of species. Cardiac auscultation is quite difficult in these animals, so it is recommended to use non-invasive electronic equipment as the Doppler, the multiparameter monitor, the pulse oximeter and esophageal stethoscope for assistance in monitoring heart and respiratory rate, providing important information trans-operative information. The realization of amputations in crocodilians is common, however there are few reports in the literature about their anesthesia, this way, this article has a significant contribution to a better anesthetic protocol clarification to be used in these animals. Therefore, it is concluded that the anesthetic protocol based on premedication with ketamine and medetomindina, propofol induction and maintenance with isoflurane, besides local anesthesia with lidocaine was effective and safe for thoracic limb amputation surgery in an American alligator.
\end{abstract}

Keywords: restraint, surgery, reptiles, wildlife.

Descritores: contenção, cirurgia, répteis, animais silvestres.

${ }^{1}$ Programa de Pós-graduação em Medicina Veterinária, Universidade Federal do Pampa (UNIPAMPA), Uruguaiana, RS, Brazil. ${ }^{2}$ Programa de Pós-graduação em Medicina Veterinária, Universidade Federal de Santa Maria (UFSM), Santa Maria, RS. ${ }^{3}$ Programa de Pós-graduação em Medicina Veterinária, Universidade Federal do Paraná (UFPR), Curitiba, PR, Brazil. ${ }^{4}$ M.V. Autônoma, Porto Alegre, RS, Brazil. CORRESPONDENCE: R. Gorczak [rochellegorczak@gmail. com - Tel.: +55 (55) 3421-8445]. Serviço de Anestesiologia Veterinária - Hospital Universitário Veterinário, Unipampa. Campus Uruguaiana. BR 472, Km 592. CEP 97500-970 Uruguaiana, RS, Brazil. 


\section{INTRODUÇÃO}

Na classe Reptilia (répteis) não existem espécies dóceis, portanto torna-se essencial a contenção física e química nesses animais. Diante disso, é de suma importância o conhecimento da fisiologia da espécie, uma vez que esses animais são ectotérmicos e seu metabolismo dependente da temperatura, podem-se observar reações imprevisíveis à mesma droga em condições ambientais diferentes e respostas fisiológicas variadas [5].

O protocolo anestésico visa a imobilização, a analgesia e o relaxamento muscular para uma adequada anestesia. Além disso, outras características importantes incluem a realização de anestesia segura e facilmente reversível. Em répteis, a via de administração dos medicamentos e a temperatura corporal do animal são fatores importantes na ação dos anestésicos [3]. Um dos procedimentos mais realizados nesses animais é a amputação de membro, principalmente por lesões ocasionadas por brigas no período reprodutivo [1]. Poucos relatos foram descritos sobre anestesia nestes animais, o que torna relevante a descrição deste caso para um maior conhecimento acerca do manejo e das técnicas anestesicas para a espécie. Diante disso, o objetivo do presente estudo é relatar o protocolo anestésico utilizado e monitoração dos parâmetros vitais em um jacaré-americano submetido à cirurgia para amputação de membro.

\section{CASO}

Foi atendido um jacaré-americano (Alligator mississipiensis), fêmea, com aproximadamente oito anos de idade, dois metros de comprimento e pesando $122 \mathrm{~kg}$, com histórico de briga com outro animal há seis meses, resultando em uma ferida no membro torácico direito (MTD). Na ocasião, devido a coincidir com o período reprodutivo, optou-se por não realizar tratamento. Após esse período, no exame físico, observou-se perda do movimento e presença de ferida na extremidade do MTD.

Com o auxílio do condicionamento, foi feita contenção física da paciente para realização do exame radiográfico do membro com alteração, o qual se evidenciou proliferação óssea, irregularidade da superfície articular, principalmente lise óssea, erosão do osso subcondral e pericondral na epífise distal do úmero e proximal de ulna e rádio, sendo essa última de maior acometimento. Ainda, também foi verificado, área radiolucente em diáfise média de úmero com es- trutura radiopaca adjacente compatível com fragmento ósseo, diminuição do espaço articular, e presença de reação periosteal em diáfise médio-proximal de ulna e médio distal de úmero, sendo esses achados sugestivos de osteomielite e artrite séptica (Figura 1). Devido à gravidade das lesões e perda da função do membro, optou-se pela amputação do mesmo.

Para anestesia e cirurgia, a contenção do animal foi feita com fitas junto a um tablado. Como medicação pré-anestésica, foi utilizada a associação de cetamina (Ketamine $\left.\mathrm{HClCIII}{ }^{\circledR}\right)^{1}$, na dose de $10 \mathrm{mg} / \mathrm{kg}$ e medetominida (Medetomidine $\mathrm{HCl} \AA)^{1}$, na dose de $0,1 \mathrm{mg} / \mathrm{kg}$, ambos por via intramuscular. Após 30 min, para indução, utilizou-se propofol (PropoFlo®) ${ }^{2}$, na dose de $4 \mathrm{mg} / \mathrm{kg}$ pela via intravenosa utilizando a veia coccígea lateral (Figura 2). Após 15 min, com o auxílio de uma madeira fazendo um sistema de alavanca para abertura da cavidade oral, foi realizada a intubação utilizando sonda endotraqueal número 11 sem inflar o cuffing. Na manutenção foi empregado isofluorano (Isoflurane $\left.{ }^{\circledR}\right)^{2} 2,5 \%$ vaporizado em oxigênio a $100 \%$, em circuito semi-fechado, nos primeiros $20 \mathrm{~min}$, e $1 \%$ até o final do procedimento.

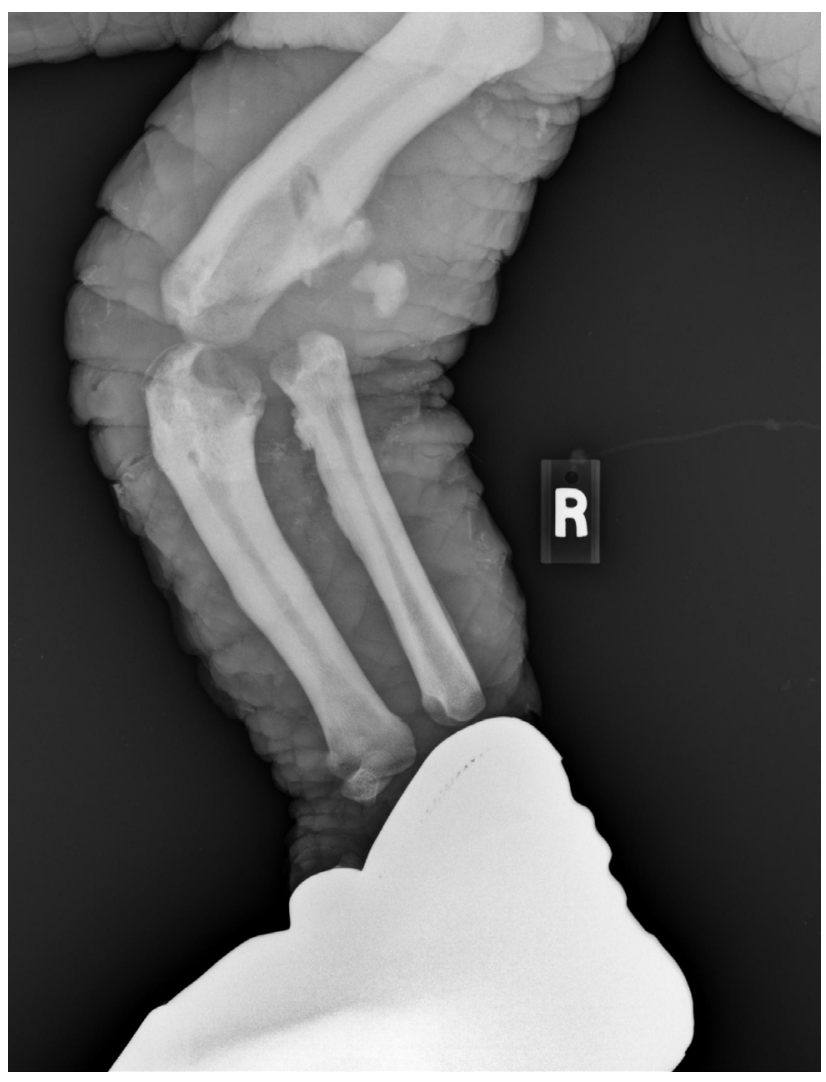

Figura 1. Radiografia do membro torácico direito em Jacaréamericano (Alligator mississipiensis). Observa-se proliferação óssea, irregularidade da superfície articular, principalmente lise óssea, erosão do osso subcondral e pericondral na epífise distal do úmero e proximal de ulna e rádio. 


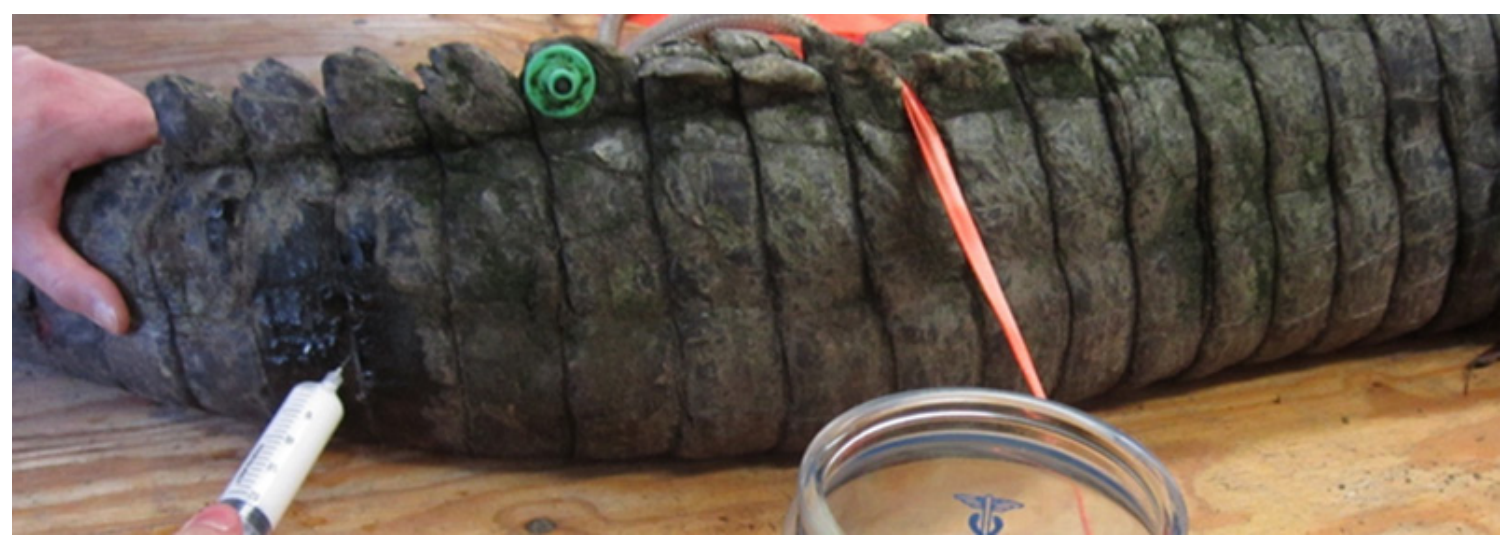

Figura 2. Administração de propofol pela via intravenosa na veia coccígea lateral em Jacaré-americano (Alligatormississipiensis).

A frequência cardíaca foi avaliada por meio do aparelho Doppler com a probe posicionada acima da artéria óptica, e a frequência respiratória pela observação visual e do balão no sistema circular de oxigênio. Os demais parâmetros foram mensurados utilizando um sensor do monitor multiparamétrico conectado à língua (Figura 3).

O jacaré foi posicionado em decúbito ventral. Realizou-se bloqueio anestésico local próximo à linha de incisão, no terço médio do úmero, com $2 \mathrm{mg} / \mathrm{kg}$ de lidocaína $2 \%$ sem vasoconstritor (Lidocaine $\left.\mathrm{HCl}{ }^{\circledR}\right)^{2}$, diluída em $\mathrm{NaCl}$ 0,9\% até completar o volume de 10 $\mathrm{mL}$, para auxiliar na analgesia. Enquanto aguardava-se dez minutos para início da cirurgia, procedeu-se a antissepsia com $\mathrm{NaCl}$ 0,9\%, iodopovidine e álcool iodado.

Os parâmetros registrados durante o procedimento mantiveram-se dentro do normal do padrão da espécie, ou seja, três movimentos respiratórios por min e 30 batimentos cardíacos por min e a temperatura permaneceu $27^{\circ} \mathrm{C}$. A duração do procedimento cirúrgico foi de $80 \mathrm{~min}$, com um consumo total de $80 \mathrm{~mL}$ de isofluorano. Ao final do procedimento o paciente foi mantido intubado recebendo oxigenioterapia por $15 \mathrm{~min}$.

Após o procedimento cirúrgico foi administrado meloxicam (Meloxicam SR $)^{1}$, na dose de 0,2 $\mathrm{mg} / \mathrm{kg}$ e o reversor atipamezole (Atipamezole $\left.\mathrm{HCl}{ }^{\circledR}\right)^{1}$, na dose de $0,5 \mathrm{mg} / \mathrm{kg}$ ambos pela via intramuscular. $\mathrm{O}$ animal levou cerca de $7 \mathrm{~h}$ para retornar completamente da anestesia e novamente liberado no recinto.

\section{DISCUSSÃO}

Diferentes protocolos anestésicos podem ser empregados para crocodilianos, utilizando tanto anestesia local como geral. No caso relatado, como medicação pré-anestésica, foi administrado cetamina e medetominida, sendo a cetamina um dissociativo que pode ser aplicado pela via venosa ou intramuscular com muita segurança [6]. Para indução anestésica, alguns autores sugerem o uso do propofol intravenoso, em animais menores, pela veia coccígea ventral, e nos maiores, pela veia coccígea lateral [7], como foi utilizado no paciente em questão. Esse fármaco promove efeito anestésico com duração média de 30 min nesses animais, tempo suficiente para realização de diversos procedimentos e intubação endotraqueal, assim como observado no presente relato.

A anestesia local deve proporcionar paralisia reversível dos nervos sensoriais, mínima toxicidade local e sistêmica e ser absorvido lentamente, promovendo prolongada anestesia local [1,7]. Como a pele de répteis é sensível ao estímulo doloroso [8], a realização do bloqueio anestésico com lidocaína $2 \%$ sem vasoconstritor foi benéfica e, possivelmente, reduziu o volume de isofluorano consumido. Portanto, a dose de $2 \mathrm{mg} / \mathrm{kg}$ de lidocaína diluída em $\mathrm{NaCl}$ 0,9\% para realização do

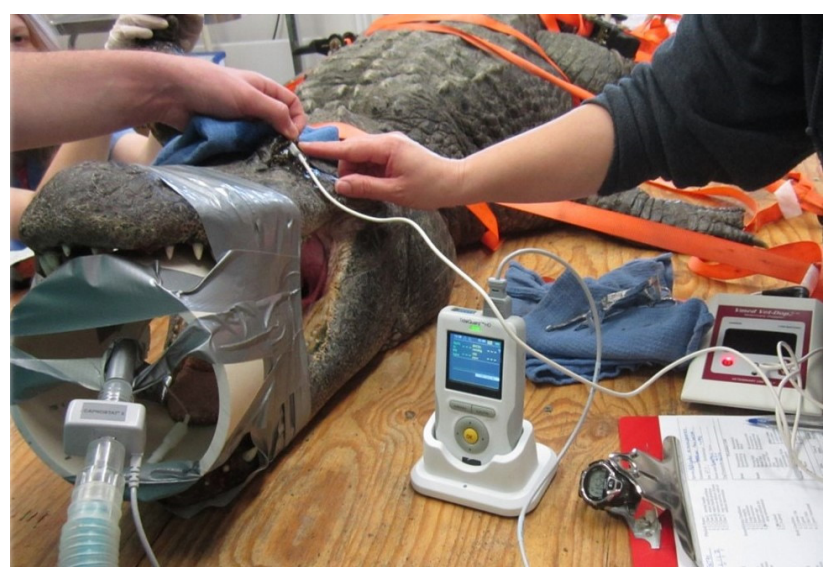

Figura 3. Monitoração trans-anestésica utilizando aparelho Doppler e monitor multiparamétrico em Jacaré-americano (Alligator mississipiensis). 
bloqueio regional foi eficiente para a espécie, não sendo necessário utilizar a dose máxima de $5 \mathrm{mg} / \mathrm{kg}$ [1].

A auscultação dos batimentos cardíacos de répteis pode ser difícil utilizando apenas o estetoscópio, por isso, assim como realizado no animal em questão, recomenda-se a utilização de equipamentos eletrônicos de amplificação, como o Doppler, que pode ser colocado sobre o coração ou região cervical ventral, para observar o fluxo sanguíneo por meio de sinais sonoros, e, no caso descrito, a probe foi posicionada acima da artéria óptica, tendo ótima repercussão sonora. Também pode ser utilizado oxímetro de pulso, na cloaca ou na língua, para mensurar a saturação da hemoglobina. Além disso, o monitor cardíaco ou estetoscópio esofágico pode ser utilizado para auxiliar na monitoração da frequência cardíaca e respiratória [4]. Esses são métodos não invasivos de monitoramento, que proporcionam importantes dados trans-operatórios [11,12].

Em um estudo realizado com quatro crocodilos, observou-se que a administração de atipamazole para reversão dos efeitos da medetomidina após anestesia, minimizou o período do retorno anestésico [10], assim como observado no presente relato.

Em casos cirúrgicos, almofadas térmicas, bolsas de água quente ou qualquer outra fonte de calor deve ser fornecida ao paciente, a fim de manter seu metabolismo elevado. Esse procedimento facilita a eliminação de drogas, consequentemente acelera a recuperação. Assim como no caso em questão, a indução, a manutenção e a recuperação foram feitas em um ambiente dentro da variação de temperatura ideal para a espécie $\left(26^{\circ} \mathrm{C}\right.$ a $\left.32^{\circ} \mathrm{C}\right)$ [9]. Se a temperatura permanecer inferior a ideal para espécie, o tempo de indução e retorno anestésico podem se tornar mais prolongados. Portanto, recomenda-se a internação para monitoração da temperatura pós-cirúrgica, para minimizar os distúrbios fisiológicos e facilitar a recuperação [4,2], nesse caso o paciente não permaneceu internado, pois além da temperatura ambiente estar adequada, o mesmo era considerado perigoso para manipulação.

Embora seja comum esse tipo de procedimento em crocodilianos, poucos relatos existem na literatura sobre a anestesia dos mesmos, dessa forma, este artigo possui uma contribuição significativa para melhor esclarecimento do protocolo anestésico a ser utilizado nesses animais. Portanto, conclui-se que o protocolo anestésico baseado na pré-medicação com cetamina e medetominida, indução com propofol e manutenção com isofluorano, além de anestesia local com lidocaína, foi eficiente e seguro para cirurgia de amputação de membro torácico em jacaré americano.

\section{MANUFACTURERS}

${ }^{1}$ ZooPharm. Laramie, WY, USA.

${ }^{2}$ Abbott Laboratories. Abbott Park, IL, USA.

Declaration of interest. The authors report no conflicts of interest. The authors alone are responsible for the content and writing of the paper.

\section{REFERENCES}

1 Bassetti L.A.B. \& Verdade L.M. 2014. Crocodylia (Jacarés e Crocodilos). In: Cubas Z.S., Silva J.C.R. \& Catão-Dias J.L. (Eds).Tratado de Animais Selvagens Medicina Veterinária. v.1. 2.ed. São Paulo: Roca, pp.154-169.

2 Cavalcanti R.L., Crosignani N., Mucillo M., Stedile R., Alievi M., Beck C.A., Schiochet F., Nóbrega F. \& Rocha J.P. 2007. Protocolo anestésico em tartaruga cabeçuda (Caretta caretta) para celioscopia exploratória. Acta Scientiae Veterinariae. 35(Suppl 2): s311-s312.

3 Cubas P.H. \& Baptistotte C. 2007. Chelonia (Tartaruga, Cágado, Jabuti). In: Cubas Z.S., Silva J.C.R. \& Catão-Dias J.L. (Eds). Tratado de Animais Selvagens. São Paulo: Roca, pp. 86-119.

4 Fleming G.J. 2007. Crocodilians (Crocodiles, Alliggators, Caimans, Gharial). In: West G. Heard D. \& Caulkett N. (Eds). Zoo Animal \& Wildlife Immobilization and Anesthesia. Ames: Blackwell Publishing, pp.223-232.

5 Frye F.L. 2007. Condições Patológicas Relacionadas ao Ambiente de Cativeiro. In: Anais III Jornada Grupo Fowler: Encontro Nacional de Medicina de Répteis (Curitiba, Brasil).pp.13-42.

6 Guzmeli C.A.P., Rodríguez G.S., López H.A.D., Fleitas E.P., Marchena L.G., Targarona R.R., Pérez M.S., García A.C. \& Sosa V.M.R. 2014. Efectividad de la ketamina como anestésico general en ejemplares jóvenes deCrocodylus rhombifer. Revista electrónica de Veterinária. 15(3): 1-13.

7 Hatt J. \& Jurato O.M. 2012. Depth of Anesthesia Monitoring by Bispectral Analysis in Zoo Animals. In: Miller R.E. \& Fowler M. (Eds). Zoo and Wild Animal Medicine: Current Therapy. 7th edn. St. Louis: Elsevier Saunders, pp.147- 152. 8 Lock B.A. \& Wellehan J. 2015. Ophidia (Snakes). In: Fowler M.E. \& Miller R.E. (Eds). Fowler's Zoo and Wild Animal Medicine. 8th edn. St. Louis: Saunders, pp.61-74. 
R. Gorczak, R.O. Chaves, M.L. Teixeira, et al. 2017. Anestesia em Jacaré-americano (Alligator mississipiensis) para Amputação de Membro.

9 Oliveira P.M. 2003. Répteis. In: Animais Silvestres e Exóticos na Clínica Particular. São Paulo: Roca, pp.197-246.

10 Olsson A. \& Phalen D. 2012. Medetomidineimmobilisation and atipamezole reversal in large estuarine crocodiles (Crocodylus porosus) using metabolically scaled dosages. Australian Veterinary Journal. 90(6): 240-244.

11 Olsson A. \& Phalen D. 2013. Comparison of biochemical stress indicators in juvenile captive estuarine crocodiles (Crocodylus porosus) following physical restraint or chemical restraint by midazolam injection. Journal of Wildlife Diseases. 49(3): 560-567.

12 Píparo L.J. 2007. Particularidades Cirúrgicas em Répteis. In: Anais III Jornada Grupo Fowler: Encontro Nacional de Medicina de Répteis. (Curitiba, Brasil). pp.195-208. 